\title{
O Papel da Atenção na Manutenção da Informação Visuoespacial na Memória Operacional
}

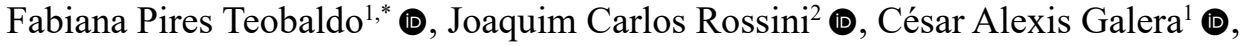 \\ \& Jeanny Joana Rodrigues Alves de Santana ${ }^{2} \oplus$ \\ ${ }^{1}$ Universidade de São Paulo, Ribeirão Preto, SP, Brasil \\ ${ }^{2}$ Universidade Federal de Uberlândia, Uberlândia, $M G$, Brasil
}

\begin{abstract}
RESUMO - A memória operacional ainda apresenta várias questões pouco compreendidas. O presente estudo teve como objetivo investigar a eficiência de retrodicas atentivas no processo de manutenção da informação visuoespacial na memória operacional. Foram delineados dois experimentos, definidos por uma tarefa única (memorização) ou dupla (memorização e busca visual). Os resultados sugerem que o efeito da retrodica informativa proporcionou uma melhora na probabilidade de recuperação da informação memorizada na presença ou na ausência da tarefa de busca visual, mesmo em condições que a busca era iniciada $50 \mathrm{~ms}$ após a apresentação da retrodica. Esse resultado é discutido em termos de um processo de múltiplas etapas no processo de alocação da atenção na memória operacional visual.
\end{abstract}

PALAVRAS-CHAVE: memória operacional, retrodica, atenção, visuoespacial

\section{The Role of Attention on the Information Maintenance in Visuo-Spatial Working Memory}

\begin{abstract}
The study of the visual working memory still presents several issues poorly understood. The present study aimed to investigate the efficiency of attentive retrocues in the process of maintenance of visuospatial information in working memory. For that, two experiments defined by a task of memorization and retrieval of visuospatial information with the aid of retrocue were conducted. The results suggest that the effect of the valid retrocue provided better accuracy in recovering the visuospatial information on the presence or absence of a visual search task. This result is discussed in terms of the existence of multiple attentional components in the visual working memory.
\end{abstract}

KEYWORDS: working memory, retrocue, attention, visuospatial

\section{INTRODUÇÃO}

A capacidade de codificar, manter e recordar informações por um breve intervalo de tempo é fundamental para a interação comportamental com o meio circundante. Essa capacidade é atribuída a um conjunto de sistemas cognitivos definidos como Memória Operacional. Um dos modelos multimodais mais influentes de memória operacional foi proposto inicialmente por Alan Baddeley e colaboradores (Baddeley \& Hitch, 1974; Baddeley, 1986; Baddeley, 2000; Baddeley, 2007) e propõe que a codificação, manutenção e recuperação da informação processada em um curto intervalo de tempo são possíveis por meio de um sistema composto por três subsistemas de armazenamento da informação, interdependentes e modulares, coordenados por um sistema atentivo, conhecido como sistema executivo central. Os três subsistemas de armazenamento envolvem um reverberador fonológico conhecido como alça fonológica (phonological loop) que seria responsável pela manutenção e reverberação da informação fonética, um subsistema de armazenamento

*E-mail: fpiresteobaldo@yahoo.com.br

- Submetido: 26/01/2017; Revisado: 20/02/2019; Aceito: 16/01/2020. 
visuoespacial conhecido como esboço visuoespacial (visuospatial sketch-pad) que seria responsável pela manutenção ativa da informação visuoespacial e integração da informação visual em representações unitária, bem como pela geração de imagens mentais visuais. Um terceiro subsistema de armazenagem seria responsável pela manutenção simultânea de informações multimodais que caracterizam um episódio. Esse subsistema é conhecido como buffer episódico (episodic buffer). Esses três subsistemas seriam gerenciados por um sistema executivo que apresentaria quatro características fundamentais: a capacidade de focalização dos recursos atentivos, a divisão dos recursos atentivos entre duas tarefas, a capacidade de alternar o foco da atenção entre tarefas e um papel conectivo geral entre a memória operacional e a memória de longo prazo (Baddeley, 1996; Baddeley, 2007).

Em meados da década de 1990, Logie (1995) propôs um modelo de memória operacional visuoespacial que amplia a concepção de esboço visuoespacial inicialmente proposto. Nesse modelo, o subsistema visuoespacial seria composto por dois armazenadores, sendo um passivo, conhecido como visual cache, e um componente ativo, conhecido como inner scribe. O visual cache seria responsável pelo armazenamento da informação visual contida em uma única cena visual, como forma e cor. Além de estímulos visuais diretamente percebidos, o visual cache poderia manter representações visuais de cenas descritas verbalmente ou mesmo de estímulos explorados pelo toque, o que proporcionaria um caráter semântico a esse subsistema. Por sua vez, o inner scribe seria responsável pelo armazenamento de sequências de movimentos relacionando os estímulos visuais a suas localizações espaciais. O sistema inner scribe seria também responsável pela atualização da informação armazenada no visual cache. No modelo proposto por Logie (1995, 2011), o sistema executivo central seria responsável pelo processamento atentivo da informação memorizada e pela geração de imagens mentais visuais. Por fim, o subsistema episódico (episodic buffer) seria caracterizado como um armazenador multidimensional, responsável pela manutenção das representações multimodais e pela recuperação da informação armazenada na memória de longo prazo, integrando-as e submetendo-as à inspeção consciente.

Vários estudos indicam uma sobreposição significativa entre o sistema de memória operacional e o sistema atentivo (Myers et al., 2017; Peters et al., 2015; Souza \& Oberauer, 2016; Theeuwes et al., 2011), todavia, uma questão ainda muito investigada na literatura diz respeito ao papel dos processos atentivos na codificação e manutenção da informação na memória operacional visual. Essa relação entre o sistema atentivo e a representação mental da informação tem sido intensamente investigada após o trabalho seminal de Griffin e Nobre (2003) (ver também Landman, Spekreijse, \& Lamme, 2003) que propuseram um procedimento, conhecido como retrodica, em que os participantes eram solicitados a memorizar uma cena com estímulos visuais definidos pela conjunção de forma e cor e apresentados simultaneamente no campo visual. Depois de um intervalo de retenção de $1500-2500$ ms, uma posição do campo visual era indicada (dica retroativa ou retrodica). A posição indicada pela retrodica pode ser compatível com o estímulo a ser recuperado (retrodica informativa), incompatível ou neutra em relação ao estímulo a ser recuperado (retrodicas não informativas). Após o fim da apresentação da retrodica e de um intervalo de tempo variável, um estímulo teste era apresentado. A tarefa do participante era julgar se o estímulo teste havia sido apresentado no arranjo inicial. Em linhas gerais, os resultados desses experimentos indicam uma maior acurácia e um tempo de reação menor nas provas em que a retrodica é informativa, em comparação com as provas nas quais a retrodica é não informativa. Esse padrão de resultados sugere que a atenção visual pode ser direcionada para uma representação mental do estímulo mantido na memória operacional visuoespacial. Posteriormente, vários estudos fazendo uso desse procedimento de retrodicas corroboraram esses achados iniciais (Lepsien et al., 2005; Lepsien \& Nobre, 2006; Lepsien \& Nobre, 2007; Makovski et al., 2008; Makovski, 2012; Makovski \& Jiang, 2007; Murray et al., 2013). Uma interpretação plausível decorrente desses achados é que os recursos atentivos, alocados por meio da retrodica informativa, influenciam significativamente tanto na codificação quanto na manutenção dos estímulos visuais na memória operacional visuoespacial (Galera et al., 2016). No entanto, a natureza dos recursos atentivos mobilizados pela retrodica ainda é controversa e os resultados obtidos em alguns estudos apontam o não envolvimento de recursos atentivos na manutenção da informação visual na memória operacional visual (Hollingworth \& Maxcey-Richard, 2013). Nesse sentido, Allen et al. (2006) obtiveram resultados que sugerem uma certa independência de recursos atentivos na manutenção da informação na memória operacional visual. Esses autores propuseram uma série de experimentos que visavam investigar o efeito de uma tarefa atentiva concorrente, em contagem regressiva, realizada durante o período de retenção mnemônica de estímulos definidos por uma característica única ou estímulos definidos pela integração de um conjunto de características. $\mathrm{O}$ resultado principal dessa investigação sugere que a realização concomitante de uma tarefa atentiva não relacionada à tarefa de memorização apresenta um efeito similar, tanto na capacidade de armazenamento dos estímulos definidos por uma conjunção de características (binding condition) quanto na capacidade de armazenamento dos estímulos definidos por uma característica única (feature condition). Esse é um resultado bastante interessante, pois sugere que o armazenamento da informação visual baseado na integração de características independe de recursos atentivos.

Posteriormente, Johnson et al. (2008) investigaram o papel da atenção na manutenção da informação visual 
armazenada em termos de características isoladas e de combinação de características. Esses autores solicitaram aos participantes que realizassem uma tarefa de busca visual durante o intervalo de retenção de uma tarefa de detecção de mudança. Nesse procedimento, a tarefa de busca visual apresentava a função de remobilizar os recursos atentivos inicialmente mobilizados para a manutenção dos itens memorizados. Caso a manutenção dos itens na memória operacional compartilhasse recursos atentivos empregados na tarefa de busca, era esperado um prejuízo significativo na recuperação dos estímulos visuais definidos por uma conjunção de características. Os resultados obtidos nesse estudo sugerem que a presença da tarefa de busca visual causa um prejuízo geral, que é semelhante tanto na recuperação dos estímulos definidos por característica única quanto na recuperação dos estímulos definidos por uma conjunção de características. Também sugere que a manutenção de itens visuais apresentados simultaneamente e definidos por mais que uma característica não requer uma mobilização significativa de recursos da atenção visual, o que corrobora, em parte, os resultados observados por Allen et al. (2006).

Mais recentemente, Hollingworth e Maxcey-Richard (2013) utilizaram o procedimento de retrodicas, obtendo resultados que sugerem que a atenção sustentada não é necessária para a manutenção da informação na memória operacional visual. Nesse estudo, uma tarefa de busca visual foi inserida, em parte das provas, $500 \mathrm{~ms}$ depois que uma retrodica válida ou neutra era apresentada. A retrodica válida indicava uma das posições anteriormente ocupada pelos estímulos de memorização, enquanto a retrodica neutra indicava todas as posições previamente ocupadas. Assim, caso a atenção fosse necessária para manter ativamente o item sugerido pela retrodica na memória operacional visual, o reengajamento dos recursos atentivos na tarefa de busca visual poderia impactar negativamente no efeito da retrodica informativa. Os resultados observados nesses experimentos sugerem que, apesar de um impacto negativo na acurácia dos participantes na tarefa de recuperação da informação associado a presença da tarefa dupla, a presença da tarefa de busca visual não provoca um efeito deletério significativo na magnitude do efeito da retrodica válida na tarefa de recuperação. Em outras palavras, a magnitude do efeito da retrodica é o mesmo, independentemente da presença ou ausência da tarefa de busca visual. Baseados nesse resultado, os autores concluíram que a manutenção seletiva da informação na memória operacional parece não depender da atenção sustentada. Em um estudo subsequente, Rerko et al. (2014) corroboram e ampliam essa conclusão, investigando em três experimentos se o efeito benéfico da retrodica na recuperação da informação visual na memória operacional está associado à alocação de recursos da atenção. No primeiro experimento desse estudo, os participantes realizaram uma tarefa interveniente de classificação de cores que requeria uma remobilização da atenção para longe da representação indicada pela retrodica. No segundo e no terceiro experimentos, os autores testaram a hipótese de que o foco da atenção na memória operacional poderia ser independente do foco da atenção perceptual. Nesses experimentos, os participantes realizavam uma tarefa interveniente que exigia uma realocação de recursos entre representações na memória operacional. A conclusão geral desse estudo foi que o efeito da retrodica não é atenuado pela realocação do foco da atenção perceptual ou pela realocação de recursos da atenção entre representações na memória operacional. Baseado nesse resultado, os autores concluíram que a atenção sustentada não é necessária para manter a representação indicada pela retrodica em um estado de elevada acessibilidade. Recentemente, Myers et al. (2017) sugeriram um modelo de múltiplas etapas para a orientação, manutenção, ressignificação e recuperação da informação na memória operacional visual, que pode ampliar, de maneira significativa, a compreensão da relação entre atenção e memória operacional na interpretação de resultados que sugerem a não necessidade de recursos atentivos na manutenção da informação na memória operacional visual.

O presente estudo teve como objetivo investigar o envolvimento de recursos da atenção sustentada na manutenção da informação armazenada na memória operacional visual. Para tanto, foram delineados dois experimentos, que utilizaram um procedimento de tarefa dupla e retrodicas, similar ao utilizado por Hollingworth e Maxcey-Richard (2013), em apresentações sequenciais ou simultâneas de estímulos visuais em uma tarefa de memorização. Diferentemente do procedimento adotado por Hollingworth e Maxcey-Richard (2013), nas condições de tarefa dupla (memorização e busca visual), a tarefa de busca visual foi apresentada após um breve intervalo de $50 \mathrm{~ms}$ após a apresentação da retrodica informativa ou não informativa. No primeiro experimento, o procedimento de retrodicas e tarefa dupla (memorização serial e busca visual) ou tarefa única (memorização serial) foi utilizada com o intuito de investigar o efeito de retrodicas informativas ou não informativas em função da tarefa de busca visual e da posição temporal de apresentação sequencial dos estímulos visuais memorizados. $\mathrm{O}$ segundo experimento investigou, por meio do mesmo procedimento, um possível efeito da busca visual na eficiência da retrodica na manutenção de estímulos apresentados simultaneamente durante a tarefa de memorização. Nessa condição, a carga mnemônica foi manipulada em dois ou quatro estímulos com o objetivo de investigar o efeito da retrodica em função desse fator. Os participantes realizaram o experimento após a anuência ao Termo de Consentimento Livre e Esclarecido aprovado pelo Comitê de Ética em Pesquisa. 


\section{EXPERIMENTO ।}

O objetivo deste experimento foi investigar um possível efeito de uma tarefa dupla (busca visual) no ganho proporcionado por uma retrodica informativa no desempenho em uma tarefa de memorização visuoespacial sequencial. Para tanto, foram apresentados quatro estímulos visuais para memorização serial em quatro localizações distintas em uma área central da tela de apresentação. Após a tarefa de memorização, foi apresentada uma retrodica, que poderia ser não informativa (retrodica neutra ou inválida) ou informativa (retrodica válida), que indicava uma localização espacial ocupada por um dos estímulos a ser recuperado ao final da prova. Após um intervalo variável, em metade das provas, o participante deveria realizar uma tarefa dupla de busca visual. Em seguida, o estímulo teste era apresentado e o participante deveria realizar uma tarefa de reconhecimento espacial, ou seja, dizer se a localização do item-teste foi idêntica à observada previamente.

A hipótese subjacente a esse experimento é a de que a atenção pode ser direcionada para o conteúdo armazenado na memória operacional (assim como ocorre na percepção) e, caso o efeito benéfico da retrodica informativa na recuperação da informação seja significativamente atenuado ou eliminado na presença de uma tarefa dupla que exija a realocação da atenção, isso poderá ser considerado uma evidência de que o efeito benéfico proporcionado pela retrodica está associado aos recursos da atenção sustentada. Caso contrário, se o efeito da retrodica apresentar uma independência em relação a presença ou a ausência da tarefa dupla, isso poderá sugerir o não envolvimento da atenção sustentada nesse processo.

\section{Método}

\section{Participantes}

O Experimento 1 foi realizado por 11 participantes $(9$ do sexo feminino), com idade média de 22,64 anos ( $D P=$ $5,33)$. Todos os participantes apresentavam visão normal ou corrigida e não apresentavam nenhuma demanda clínica declarada.

\section{Material e Estímulos visuais}

A apresentação dos estímulos e o registro do tempo de reação dos participantes foram realizados pelo aplicativo E-Prime $2.0^{\circledR}$ (Schneider et al., 2008). A tarefa foi realizada em um ambiente reservado e com baixa luminosidade.

Para a tarefa de memorização, foram utilizados seis estímulos visuais $(1,40 \mathrm{~cm} \times 1,40 \mathrm{~cm})$ apresentados sequencialmente nos vértices de um quadrado imaginário com lado igual a $5 \mathrm{~cm}$ (Figura 1). O ponto de fixação era um quadrado com dimensões $1,35 \mathrm{~cm}$ e $1 \mathrm{~mm}$ de espessura, apresentado na cor cinza. A retrodica apresentava as mesmas dimensões do quadrado de fixação, porém com dois lados adjacentes realçados (espessura $2 \mathrm{~mm}$ ) e apresentados na cor preta), indicando um dos quatro quadrantes do campo visual. Os quatros lados do quadrado eram realçados na condição de retrodica neutra. Os estímulos da tarefa de busca visual eram seis sinais de soma (+), cinco distratores e um alvo, apresentados em um círculo imaginário de raio igual a 2,5 $\mathrm{cm}$. $\mathrm{O}$ alvo era definido pelo deslocamento do segmento vertical do sinal de soma para a esquerda ou para a direita em relação ao segmento horizontal do estímulo. O sinal de feedback para a tarefa de busca e a tarefa de memorização era o mesmo quadrado de fixação apresentado na cor azul, para acertos, e na cor vermelha, para erros.

\section{Procedimento}

O participante iniciava cada prova pressionando a tecla de espaço do teclado. Após o início de cada prova, eram apresentados três dígitos aleatórios que deveriam ser repetidos em voz alta pelo participante, um por segundo, durante toda a prova experimental (supressão articulatória). Após duas repetições bem-sucedidas, o participante pressionava novamente a barra de espaço, dando início à prova experimental. Um pequeno quadrado para a fixação ocular apresentado na cor cinza claro $(1,35 \mathrm{~cm}$ de lado) era apresentado no centro da tela de apresentação. Esse estímulo de fixação era visível até a conclusão da prova. Após 500 $\mathrm{ms}$, um estímulo visual de memorização era apresentado em um dos vértices de um quadrado imaginário $(5 \mathrm{~cm}$ de lado) por $250 \mathrm{~ms}$. Após essa apresentação e um intervalo de $250 \mathrm{~ms}$, um novo estímulo visual era apresentado em um vértice diferente do quadrado imaginário, dando sequência à apresentação temporal de quatro estímulos de memorização. Após a apresentação do último estímulo da sequência de memorização e de um intervalo de $250 \mathrm{~ms}$, dois lados adjacentes do quadrado de fixação eram realçados por 500 ms. O vértice composto por esses lados adjacentes realçados indicava a posição do estímulo relevante, que poderia coincidir ou não com o estímulo teste. Essa retrodica era informativa quando indicava a posição ocupada pelo estímulo relevante para a tarefa de memorização, ou não informativa quando indicava uma posição irrelevante (retrodica inválida) ou todas as posições dos estímulos de memorização (retrodica neutra). Em 50\% das provas e após um intervalo de $50 \mathrm{~ms}$ ou $500 \mathrm{~ms}$ do final da apresentação da retrodica, uma tarefa de busca visual era apresentada. Nas provas em que a tarefa de busca visual não foi apresentada, apenas o quadrado de fixação permanecia visível por $2.000 \mathrm{~ms}$.

Para a realização da tarefa de busca visual, o participante foi instruído a identificar se o segmento vertical que compunha o estímulo de busca estava deslocado à direita ou à esquerda do ponto central do segmento horizontal do estímulo, pressionando o mais rápido possível a tecla 


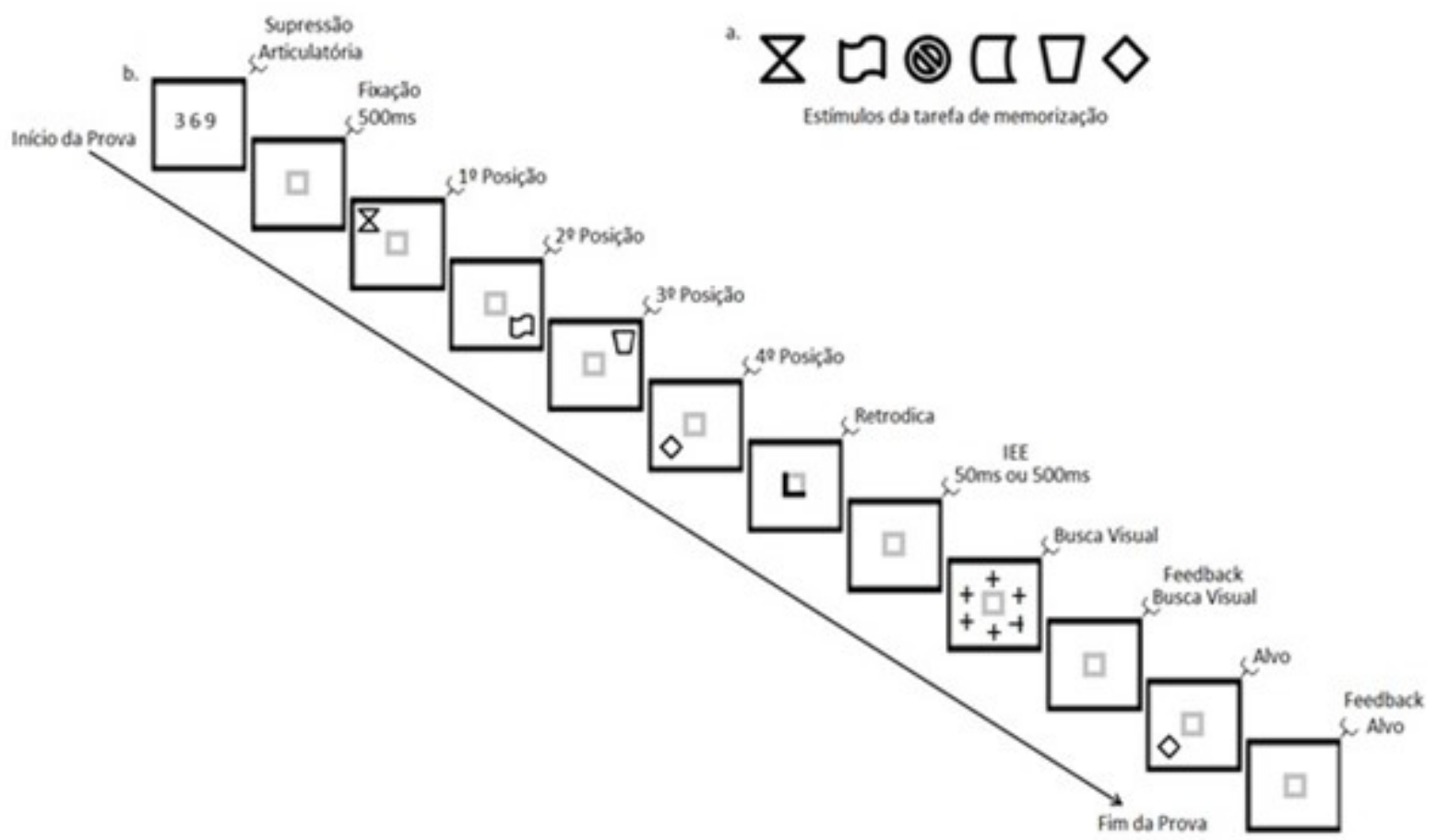

Figura 1. Representação esquemática da sequência de eventos em uma prova do Experimento 1

Nota. a. Formas geométricas utilizadas na tarefa de memorização. b. Sequência de eventos em uma prova com a apresentação da tarefa de busca visual (tarefa dupla). O sinal de feedback era apresentado na cor azul (resposta correta) ou vermelha (resposta incorreta).

1 do teclado numérico para alvo com segmento vertical deslocado à direita ou a tecla 2 para alvo com segmento vertical deslocado à esquerda. A tarefa de busca era encerrada com a resposta do participante ou após 2.000 ms. Imediatamente após o encerramento da busca visual, um sinal referente à precisão do participante na tarefa de busca era apresentado por $250 \mathrm{~ms}$, seguido pela apresentação do estímulo teste apresentado em uma das quatro posições inicialmente memorizadas. Esse estímulo permanecia visível até a resposta do participante. A tarefa do participante era responder se o estímulo teste havia sido apresentado naquela posição espacial, pressionando a tecla 1 , ou se o estímulo não havia sido apresentado naquela posição, pressionado a tecla 2 . O estímulo teste na tarefa de recuperação sempre repetia um dos estímulos inicialmente memorizados, porém esse estímulo poderia ser apresentado em uma posição diferente da apresentada inicialmente, caracterizando uma recombinação de características visuais e espaciais previamente apresentadas. Após a sua resposta, o participante era informado sobre a sua precisão na tarefa de recuperação por um sinal de feedback $(250 \mathrm{~ms})$ e a prova era encerrada juntamente com a recitação dos números apresentados no início da prova (Figura 1).

Os participantes realizaram um total de 384 provas, sendo 192 provas com a realização da tarefa de busca visual (tarefa dupla) e 192 provas sem a apresentação da tarefa de busca (tarefa única de memorização). As provas eram apresentadas em três blocos de 128 provas cada. Os participantes realizaram um treino no início da seção experimental composto por 10 provas.

\section{Resultados}

A porcentagem de acertos dos participantes na tarefa de memorização foi analisada por meio do teste ANOVA para medidas repetidas, para os fatores: (a) tipo de retrodica (informativa e não informativa); (b) tipo de tarefa (tarefa dupla $50 \mathrm{~ms}$; tarefa dupla $500 \mathrm{~ms}$; tarefa única de memorização); e (c) posição serial (posições iniciais; posição final). Essa análise confirmou um efeito significativo para os três fatores investigados: tipo de retrodica $F(1,10)=13,562$; $p<0,001 ; \eta_{\mathrm{p}}^{2}=0,57$ (retrodica informativa $=82,15 \% ; D P$ $=5,78$; retrodica não informativa $=78,29 \% ; D P=4,6$ ); tipo de tarefa $F(2,20)=10,647 ; p<0,001 ; \eta_{\mathrm{p}}^{2}=0,51$ (tarefa dupla $50 \mathrm{~ms}=75,37 \%$; $D P=4,6$; tarefa dupla $500 \mathrm{~ms}=$ $81,72 \% ; D P=3,9$; tarefa única de memorização $=83,58 \%$; $D P=4,2)$; e posição serial $F(1,10)=4,761 ; p=0,054 ; \eta_{\mathrm{p}}^{2}=$ 0,32 (posições iniciais $=77,54 \% ; D P=4,9$; posição final $=82,9 \% ; D P=4,6)$. Não houve interação significativa entre os fatores. A porcentagem de acertos na recuperação da informação memorizada nas condições investigadas é representada na Figura 2. Uma análise geral do efeito da retrodica (retrodica informativa - retrodica não informativa) 

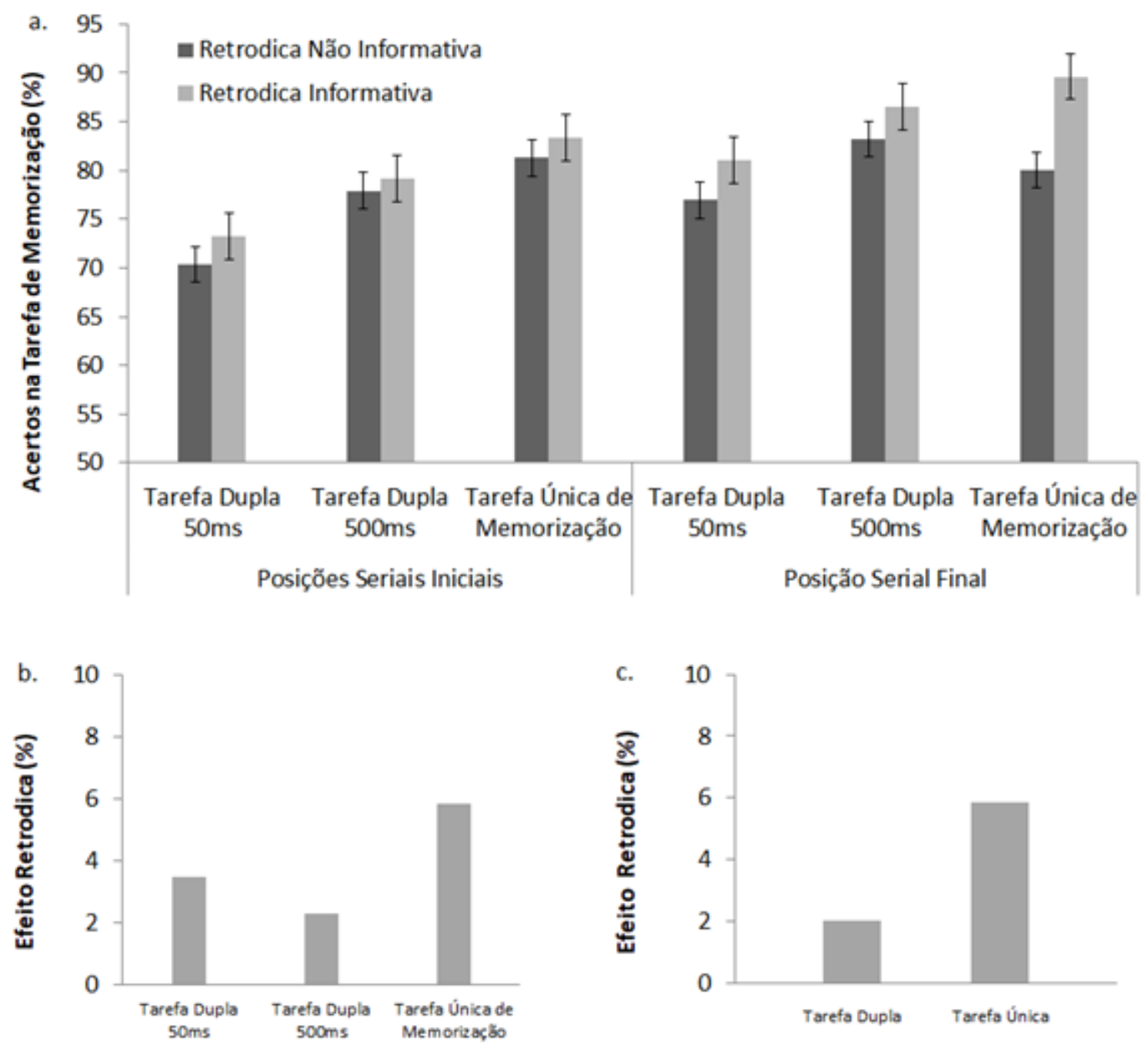

Figura 2. Resultados obtidos no Experimento 1 em função da posição da sequência de estímulos memorizados e do tipo de tarefa

Nota. a. Porcentagem de respostas corretas em função da posição da sequência de estímulos memorizados (posições iniciais e posição final) e tipo de tarefa (dupla ou única de memorização). b. Efeito da retrodica em função da tarefa (tarefa dupla 50; tarefa dupla 500; tarefa única de memorização). c. Efeito geral da retrodica (tarefa dupla e tarefa única).

não confirmou uma diferença significativa na recuperação da informação memorizada em tarefas duplas e tarefa única de memorização $F(1,10)=2,304 ; p=0,16$ (tarefa dupla $=2,87 \%$; tarefa única de memorização $=5,85 \%)$. O efeito da tarefa dupla $50 \mathrm{~ms}$ e $500 \mathrm{~ms}$ sobre a retrodica não foi significativo, respectivamente, $F(1,10)=1,844 ; p=0,20$ (tarefa dupla $50 \mathrm{~ms}=3,47 \%$; tarefa única $=5,85 \%$ ) e $F$ $(1,10)=1,356 ; p=0,27$ (tarefa dupla $500 \mathrm{~ms}=2,27 \%$; tarefa única $=5,85 \%$ ).

\section{Discussão}

Os resultados observados no Experimento 1 evidenciaram uma variação significativa na porcentagem de acertos em função de dois dos três fatores principais investigados. $\mathrm{O}$ fator retrodica foi significativo e evidenciou uma maior probabilidade de recuperação da informação memorizada nas condições de retrodica informativa, o que corrobora os resultados observados em diversos estudos (Griffin \& Nobre, 2003; Murray et al., 2013; van Moorselaar et al., 2015; Wang et al., 2017). O fator tipo de tarefa (dupla ou única) foi significativo e confirma uma redução da probabilidade de recuperação da informação na tarefa dupla. Esse resultado corrobora os achados de Hollingworth e Maxcey-Richard (2013). O fator posição serial apresentou uma significância estatística limítrofe $(p=0,054)$ e sugere que o estímulo teste da tarefa de memorização pode ser melhor recuperado quando apresentado na última posição de memorização $(82,9 \%)$ do que quando apresentado nas posições iniciais (77,54 \%) (Allen et al., 2014). Não foi confirmada uma interação significativa entre os fatores tipo de retrodica e tipo de tarefa, sugerindo que o efeito informativo da retrodica não é significativamente influenciado pela tarefa dupla (presença da tarefa de busca visual), o que corrobora 
o estudo de Hollingworth e Maxcey-Richard (2013). O presente resultado amplia os achados anteriores, uma vez que a manutenção do efeito benéfico da retrodica informativa foi observado na condição de tarefa dupla após $50 \mathrm{~ms}$. A análise realizada não confirmou uma diferença significativa do efeito da retrodica entre a condição de tarefa dupla e a condição de tarefa única de memorização. Esse resultado sugere que, após um intervalo de $50 \mathrm{~ms}$, a informação proporcionada pela retrodica está suficientemente consolidada e independe de recursos atentivos de manutenção. No presente experimento, a retrodica foi apresentada por $500 \mathrm{~ms}$ seguida de um intervalo de $50 \mathrm{~ms}$, totalizando $550 \mathrm{~ms}$ do início da apresentação da retrodica até o início da tarefa de busca visual, tempo suficiente para a orientação dos recursos atentivos para uma área pertinente da representação mental (aproximadamente $300 \mathrm{~ms}$ para a orientação endógena efetiva dos recursos atentivos) (Chakravarthi \& VanRullen, 2011; Carlson et al., 2006; Tanoue \& Berryhill, 2012). Após esse processo de orientação, a mobilização de recursos atentivos de manutenção seria desnecessária (Hollingworth \& Maxcey-Richard, 2013; Myers et al., 2017; Muhle-Karbe et al., 2017).

\section{EXPERIMENTO 2}

No Experimento 2, aprofundamos a investigação acerca do efeito de retrodica na memória operacional visual empregando o mesmo procedimento utilizado no Experimento 1. Nosso objetivo foi investigar um possível benefício proporcionado pela retrodica na recuperação da informação visual memorizada. Nesse experimento, a informação a ser memorizada foi apresentada simultaneamente. Como no primeiro experimento, uma tarefa de busca visual foi realizada em $50 \%$ das provas. Quando presente, a tarefa de busca tinha início $50 \mathrm{~ms}$ ou $500 \mathrm{~ms}$ após a apresentação de uma retrodica informativa ou não informativa quanto à tarefa de memorização. Um terceiro fator investigado foi o número de estímulos a serem memorizados (carga mnemônica: 2 ou 4 estímulos).

\section{Método}

\section{Participantes}

O Experimento 2 foi realizado por 13 participantes $(9$ do sexo feminino), com idade média de 20,69 anos ( $D P=$ $5,07)$. Todos os participantes apresentavam visão normal ou corrigida e não apresentavam nenhuma demanda clínica declarada. Dois participantes do sexo feminino também realizaram o Experimento 1.

\section{Material e Estímulos visuais}

O material e os estímulos visuais utilizados no segundo experimento foram os mesmos utilizados no primeiro experimento.

\section{Procedimento}

A Figura 3 exemplifica a sucessão de eventos em uma prova do Experimento 2. Cada prova era iniciada com a apresentação de três dígitos aleatórios no centro da tela de apresentação. Os participantes eram orientados a repetir em voz alta cada dígito, na razão de um dígito por segundo, até o final da prova. Após duas repetições bem-sucedidas, a prova experimental era iniciada com a apresentação de um quadrado de fixação no centro do campo visual e que permanecia exposto até o final da prova. Após $500 \mathrm{~ms}$, um arranjo visual de dois ou quatro estímulos de memorização era apresentado por $750 \mathrm{~ms}$, seguido de um intervalo entre estímulos de 2.000 ms. Após esse intervalo, dois lados adjacentes do quadrado de fixação eram realçados (retrodica) por $250 \mathrm{~ms}$. O vértice composto por esses lados adjacentes indicava uma posição relevante a ser recuperada posteriormente. Essa retrodica era informativa quando indicava um estímulo válido de recuperação (retrodica informativa). As retrodicas não informativas indicavam um estímulo diferente do estímulo de recuperação (retrodica inválida) ou indicavam todos os quadrantes como relevantes (retrodica neutra). Em 50\% das provas e após um intervalo de $50 \mathrm{~ms}$ ou $500 \mathrm{~ms}$ da apresentação da retrodica, uma tarefa de busca visual era apresentada. Nas provas em que a tarefa de busca visual não foi apresentada, apenas o quadrado de fixação permanecia visível por $2.500 \mathrm{~ms}$. A tarefa de busca era a mesma utilizada no primeiro experimento e era encerrada com a resposta do participante ou após $2.500 \mathrm{~ms}$. Imediatamente após o encerramento da tarefa de busca visual, um sinal referente à precisão do participante na tarefa de busca era apresentado por $1.500 \mathrm{~ms}$. Imediatamente após esse sinal de feedback e um intervalo de $50 \mathrm{~ms}$, um estímulo teste de memorização era apresentado no centro da tela. A tarefa do participante era responder se o estímulo teste havia sido apresentado, pressionando a tecla 1 , ou se o estímulo não havia sido apresentado, pressionando a tecla 2. Após a sua resposta, o participante era informado sobre a sua precisão na tarefa de recuperação por um sinal de feedback $(1.500 \mathrm{~ms})$ e a prova era encerrada. Diferentemente do primeiro experimento, o estímulo visual teste da tarefa de memorização era sempre apresentado no centro da tela. Por fim, um novo estímulo foi inserido nas provas negativas, ou seja, o estímulo teste da tarefa de memorização não fazia parte do arranjo de estímulos inicialmente memorizados. No total, os participantes realizaram 288 provas divididas em dois blocos, cada um com 144 provas, das quais 96 provas com retrodica informativa e 192 provas com retrodica não informativa ( 96 provas com retrodica inválida e 96 provas com retrodica neutra). 


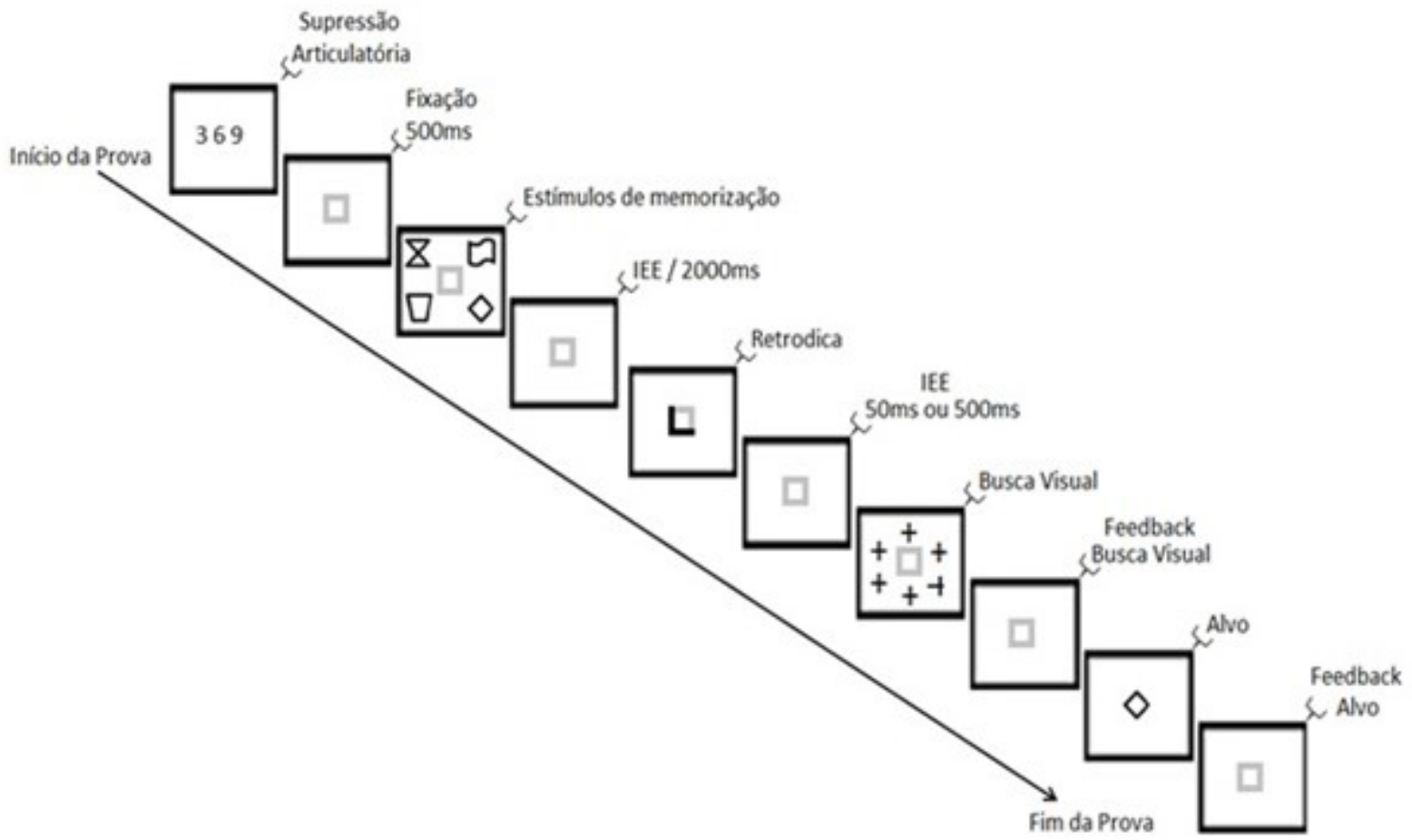

Figura 3. Representação esquemática da sequência de eventos em uma prova do Experimento 2 (Carga mnemônica 4) Nota. $O$ sinal de feedback era apresentado na cor azul (resposta correta) ou vermelha (resposta incorreta).

\section{Resultados}

A porcentagem de acertos dos participantes na tarefa de memorização foi analisada por meio do teste ANOVA para medidas repetidas, para os fatores: (a) tipo de retrodica (informativa, não informativa); (b) carga mnemônica ( 2 ou 4); (c) tipo de tarefa (tarefa dupla $50 \mathrm{~ms}$; tarefa dupla $500 \mathrm{~ms}$; tarefa única de memorização). Essa análise confirmou um efeito significativo para o fator tipo de retrodica $F(1,12)=$ 15,$95 ; p<0,001, \eta_{\mathrm{p}}^{2}=0,57$ (retrodica informativa $=84,36 \%$; $D P=4,11$; não informativa $=72,92 \% ; D P=8,29$ ) e para o fator carga mnemônica simultânea $F(1,12)=26,38$; $p<$ 0,$001 ; \eta_{\mathrm{p}}^{2}=0,69($ carga $2=83,53 \% ; D P=4,39$; carga $4=$ $73,75 \% ; D P=6)$. O fator tipo de tarefa não apresentou significância, $F(2,24)=0,624 ; p=0,54 ; \eta_{\mathrm{p}}^{2}=0,05$, (tarefa dupla $50 \mathrm{~ms}=78,9 \%$; $D P=7,2$, tarefa dupla $500 \mathrm{~ms}=$ $77,6 \% ; D P=4,1$, tarefa única de memorização $=79,5 \%$; $D P=5,2)$. Não houve efeito significativo de interação entre os fatores analisados. A porcentagem de acertos em função das condições experimentais investigadas é apresentada na Figura 4.

$\mathrm{O}$ efeito do fator retrodica (retrodica informativa retrodica não informativa) foi analisado em função do fator tipo de tarefa. Uma análise geral do efeito não confirmou uma diferença significativa do efeito da retrodica entre as condições de tarefa dupla e tarefa única $F(1,12)=0,689$; $p=0,42$ (tarefa dupla $=12,5 \%$; tarefa única $=9,25 \%)$. O efeito da retrodica não apresentou diferença significativa em função da condição de tarefas duplas $50 \mathrm{~ms}$ e $500 \mathrm{~ms}$, respectivamente, $F(1,12)=0,052 ; p=0,82$ e $F(1,12)=$ 1,$53 ; p=0,24$.

\section{Discussão}

O resultado geral do Experimento 2 sugere que a informação proporcionada pela retrodica é preservada em condições de tarefas duplas e a necessidade de recursos atentivos de manutenção é reduzida, corroborando os resultados observados no primeiro experimento. Esse resultado vai ao encontro de outros achados na literatura, que sugerem a ocorrência de múltiplas etapas no processo de alocação da atenção na memória operacional visual (Myers et al., 2017; Wang et al., 2017) e evidenciam um envolvimento limitado de recursos atentivos na manutenção da informação visuoespacial na memória operacional. Uma interpretação plausível é que, após a finalização do processo de orientação endógena da atenção, a informação da retrodica é suficientemente estável e imune à interferência de uma demanda atentiva subsequente (Hollingworth \& Maxcey-Richard, 2013; Myers et al., 2017). 

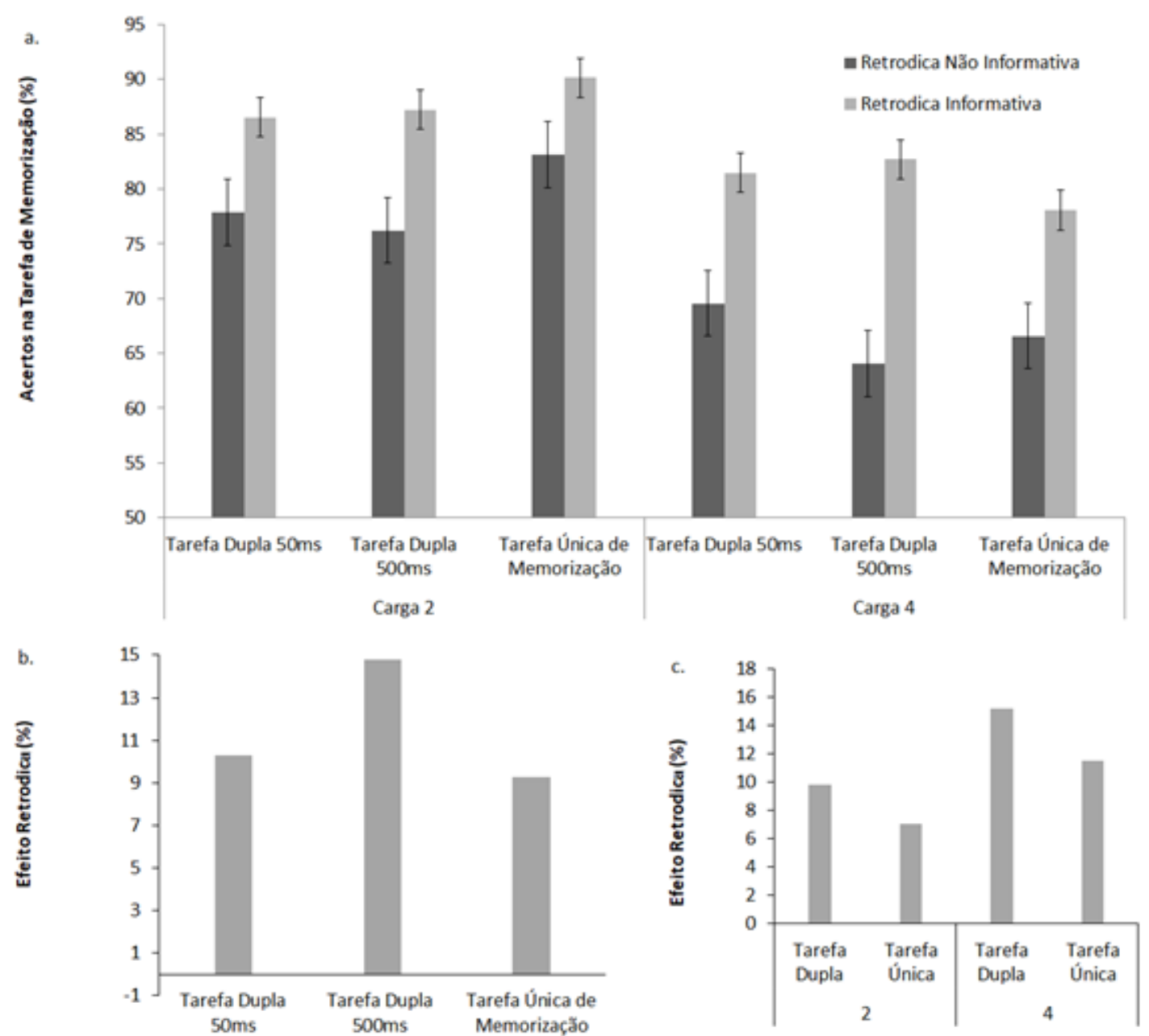

Figura 4. Resultados obtidos no Experimento 2 em função do tipo de tarefa e a carga mnemônica

Nota. a. Efeito da retrodica na porcentagem de acertos em função do tipo de tarefa (dupla 50ms; dupla 500ms; única de memorização) e da carga mnemônica (2 ou 4 estímulos simultâneos de memorização). b. Efeito da retrodica em função das tarefas (tarefa dupla 50ms; tarefa dupla 500ms; tarefa única de memorização). c. Efeito da retrodica em função da tarefa (dupla ou única) e da carga mnemônica (2 ou 4).

\section{DISCUSSÃO GERAL E CONCLUSÃO}

No presente estudo, foram investigadas questões acerca da mobilização dos recursos atentivos na memória operacional visual. O primeiro experimento investigou um possível efeito de uma tarefa de busca visual realizada logo após a memorização serial de estímulos apresentados em diferentes posições espaciais. No mesmo procedimento, foram utilizadas retrodicas visuais informativas ou não informativas, que indicavam uma posição relevante a ser recuperada. Os resultados evidenciaram um efeito geral benéfico na acurácia associado à retrodica informativa, corroborando diversos estudos anteriores (Griffin \& Nobre, 2003; Lepsien et al., 2005; Lepsien \& Nobre, 2006; Lepsien \& Nobre, 2007; Makovski, 2012; Makovski et al., 2008; Makovski \& Jiang, 2007; Murray et al., 2013;). Nos dois experimentos o efeito benéfico da retrodica informativa foi preservado em condições de tarefa dupla que demandavam uma realocação dos recursos atentivos após a apresentação da retrodica. Essa interpretação é respaldada pela confirmação de uma não interação entre os fatores tipo de tarefa e tipo de retrodica observada nos dois experimentos realizados. Interessante ressaltar que o efeito da retrodica informativa permanece preservado mesmo na condição em que a tarefa de busca visual é iniciada após $50 \mathrm{~ms}$ da conclusão da apresentação da retrodica, uma vez que, no presente estudo, a retrodica apresentou intervalos de apresentação de $500 \mathrm{~ms}$ (Experimento 1) e $250 \mathrm{~ms}$ (Experimento 2). Pode-se supor que, aproximadamente $300 \mathrm{~ms}$ após o início da apresentação da retrodica, o efeito positivo proporcionado pela retrodica é preservado, independentemente da realocação da atenção. Essa conclusão é compatível com a interpretação de que a manutenção dos estímulos na memória operacional pode ocorrer sem a mobilização de recursos atentivos, ou seja, de maneira automática livre de custo atentivo (cost-free) 
(Allen et al., 2006; Hollingworth \& Maxcey-Richard, 2013; Myers et al., 2017). De modo geral, os dois experimentos realizados apresentam evidências que sugerem a existência de múltiplos processos atentivos envolvidos na codificação e armazenamento da informação visuoespacial na memória operacional.

\section{REFERÊNCIAS}

Allen, R. J., Baddeley, A. D., \& Hitch, G. J. (2006). Is the binding of visual features in working memory resourcedemanding? Journal of Experimental Psychology: General, 135(2), 298-313. https://doi.org/10.1037/00963445.135.2.298

Allen, R. J., Baddeley, A. D., \& Hitch, G. J. (2014). Evidence for two attentional components in visual working memory. Journal of Experimental Psychology: Learning, Memory, and Cognition, 40(6), 1499-1509. https://doi.org/10.11037/xlm0000002

Baddeley, A. D., \& Hitch, G. (1974). Working memory. Psychology of Learning and Motivation, 8, 47-89. http://dx.doi. org/10.1016/S0079-7421(08)60452-1

Baddeley, A. (1986). Working memory. Oxford Psychology Series.

Baddeley, A. (1996). Exploring the central executive. The Quarterly Journal of Experimental Psychology: Section A, 49(1), 5-28.

Baddeley, A. (2000). The episodic buffer: a new component of working memory? Trends in Cognitive Sciences, 4(11), 417423. https://doi.org/10.1016/S1364-6613(00)01538-2

Baddeley, A. (2007). Working memory, thought, and action (Vol. 45). OUP Oxford.

Carlson, T. A., Hogendoorn, H., \& Verstraten, F. A. (2006). The speed of visual attention: What time is it?. Journal of Vision, 6(12), 6-6.

Chakravarthi, R., \& VanRullen, R. (2011). Bullet trains and steam engines: Exogenous attention zips but endogenous attention chugs along. Journal of Vision, 11(4), 12-12.

Galera, C., Guimarães, L. S., Rossini, J. C., \& Santana, J. J. R. A. (2016) . A recuperação da informação visual baseada na localização e nas características visuais dos objetos. Estudos de Psicologia (Natal), 21(3), 228-238. https://doi. org/10.5935/1678-4669.20160022

Griffin, I. C., \& Nobre, A. C. (2003). Orienting attention to locations in internal representations. Journal of Cognitive Neuroscience, 15(8), 1176-1194. https://doi.org/ 10.1162/089892903322598139

Hollingworth, A., \& Maxcey-Richard, A. M. (2013). Selective maintenance in visual working memory does not require sustained visual attention. Journal of Experimental Psychology: Human Perception and Performance, 39(4), 1047-1058. https://doi.org/10.1037/a0030238

Johnson, J. S., Hollingworth, A., \& Luck, S. J. (2008). The role of attention in the maintenance of feature bindings in visual short-term memory. Journal of Experimental Psychology: Human Perception and Performance, 34(1), 41-55. https:// doi.org/10.1037/0096-1523.34.1.41

Landman, R., Spekreijse, H., \& Lamme, V. A. F. (2003). Large capacity storage of integrated objects before change blindness. Vision Research, 43, 149-164. https://doi.org/10.1016/S00426989(02)00402-9

Lepsien, J., \& Nobre, A. C. (2006). Cognitive control of attention in the human brain: Insights from orienting attention to mental representations. Brain Research, 1105(1), 20-31. https://doi. org/10.1016/j.brainres.2006.03.033

Lepsien, J., \& Nobre, A. C. (2007). Attentional modulation of object representations in working memory. Cerebral Cortex, 17(9), 2072-2083. https://doi.org/10.1093/cercor/bhl116

Lepsien, J., Griffin, I. C., Devlin, J. T., \& Nobre, A. C. (2005). Directing spatial attention in mental representations: Interactions between attentional orienting and working- memory load. Neuroimage, 26(3), 733-743. https://doi. org/10.1016/j.neuroimage.2005.02.026

Logie, R. H. (1995). Visuo-spatial working memory. Erlbaum.

Logie, R. H. (2011). The functional organization and capacity limits of working memory. Current Directions in Psychological Science, 20(4), 240-245. https://doi.org/10.1177/0963721411415340

Makovski, T., \& Jiang, Y. V. (2007). Distributing versus focusing attention in visual short-term memory. Psychonomic Bulletin \& Review, 14(6), 1072-1078. https://doi.org/10.3758/ BF03193093

Makovski, T. (2012). Are multiple visual short-term memory storages necessary to explain the retro-cue effect? Psychonomic Bulletin \& Review, 19(3), 470-476. https://doi.org/10.3758/ s13423-012-0235-9

Makovski, T., Sussman, R., \& Jiang, Y. V. (2008). Orienting attention in visual working memory reduces interference from memory probes. Journal of Experimental Psychology: Learning, Memory, and Cognition, 34(2), 369-380. https:// doi.org/10.1037/0278-7393.34.2.369

Muhle-Karbe, P. S., Duncan, J., De Baene, W., Mitchell, D. J., \& Brass, M. (2017). Neural coding for instruction-based task sets in human frontoparietal and visual cortex. Cerebral Cortex, 27(3), 1891-1905.

Murray, A. M., Nobre, A. C., Clark, I. A., Cravo, A. M., \& Stokes, M. G. (2013). Attention restores discrete items to visual shortterm memory. Psychological Science, 24(4), 550-556. https:// doi.org/10.1177/0956797612457782

Myers, N. E., Stokes, M. G., \& Nobre, A. C. (2017). Prioritizing information during working memory: beyond sustained internal attention. Trends in Cognitive Sciences, 21(6), 449-461.

Peters, B., Kaiser, J., Rahm, B., \& Bledowski, C. (2015). Activity in human visual and parietal cortex reveals object-based attention in working memory. Journal of Neuroscience, 35(8), 3360-3369.

Rerko, L., Souza, A. S., \& Oberauer, K. (2014). Retro-cue benefits in working memory without sustained focal attention. Memory \& Cognition, 42, 712-728. https://doi.org/10.3758/s13421013-0392-8

Schneider, W., Eschman, A., \& Zuccolotto, A. (2008). E-Prime reference guide. Psychology Software Tools, Incorporated.

Souza, A. S., \& Oberauer, K. (2016). In search of the focus of attention in working memory: 13 years of the retro-cue effect. Attention, Perception, \& Psychophysics, 78, 1839-1860. https:// doi.org/10.3758/s13414-016-1108-5

Tanoue, R. T., \& Berryhill, M. E. (2012). The mental wormhole: internal attention shifts without regard for distance. Attention, Perception, \& Psychophysics, 74(6), 1199-1215.

Theeuwes, J., Kramer, A. F., \& Irwin, D. E. (2011). Attention on our mind: The role of spatial attention in visual working memory. Acta Psychologica, 137(2), 248-251.

van Moorselaar, D., Olivers, C. N., Theeuwes, J., Lamme, V. A., \& Sligte, I. G. (2015). Forgotten but not gone: Retro-cue costs and benefits in a double-cueing paradigm suggest multiple states in visual short-term memory. Journal of Experimental Psychology: Learning, Memory, and Cognition, 41(6), 17551763. https://doi.org/10.1037/xlm0000124

Wang, B., Yan, C., Wang, Z., Olivers, C. N., \& Theeuwes, J. (2017). Adverse orienting effects on visual working memory encoding and maintenance. Psychonomic Bulletin \& Review, 24(4), 1261-1267. 